\title{
Analysis of Coumarins by Micro High-Performance Liquid Chromatography-Mass Spectrometry with a Particle Beam Interface
}

\author{
Achille Cappiello, Giorgio Famiglini, and Filippo Mangani \\ Istituto di Scienze Chimiche, Università di Urbino, Urbino, Italy \\ Bruno Tirillini \\ Istituto di Botanica e Orto Botanico, Università di Urbino, Urbino, Italy
}

\begin{abstract}
Coumarins are a large group of compounds that are naturally present in plant tissues and that exhibit a wide range of pharmacological properties. Analytical methods based on chromatographic techniques and conventional detectors are inadequate to accurately analyze coumarins in complex matrices such as plant extracts. In this article a new method based on a modified particle beam liquid chromatography-mass spectrometry interface is described. The method allows specific and accurate determination of several coumarins in biological matrices. An application regarding the analysis of 18 coumarins in the extract of Smyrnitum perfoliatum L. is also reported. (J Am Soc Mass Spectrom 1995, 6, 132-139)
\end{abstract}

C oumarins are a large family of substances that are usually extracted from plants, where they pursue a distinct biological role: production of coumarins appears to increase when the plant becomes the object of external aggression. Most of the interest in these compounds arises from their pharmacological properties and some toxicological risks to humans. For example, scopoletin has a stabilizing effect on biological membranes with a resultant hepatoprotective activity. Scopoletin also inhibits platelet aggregation and, moreover, it blocks the neurotransmitters of the cholinergic and adrenergic neuroeffector junctions. Psolarens, another group of coumarins, are characterized by photobiological effects and are used in the photochemotherapeutic treatment (PUVA therapy) of some skin diseases with hyperproliferative conditions [1]. Unfortunately, the pharmacological effectiveness of these drugs is impaired by side effects such as severe dermatitis, erythema, phototoxicity, and possible increased risk of skin cancer, which is mainly related to the capacity of psolarens to form light-induced mono or bis adducts with DNA pyrimidine bases [2].

Mass spectrometry has shown a great potential for structure elucidation and isomer characterization of several coumarins. Different ionization techniques like electron impact (EI) [3], positive and negative chemical

Address reprint requests to Dr. Achille Cappiello, Istituto di Scienze Chimiche, Università di Urbino, Piazza Rinascimento 6, 61029 Urbino, Italy. ionization $(\mathrm{CI})[4,5]$, and electron attachment [6] have been employed successfully.

Traldi and co-workers [7-10] have investigated the structures of furocoumarin isomers, which cannot be distinguished with conventional mass spectrometric techniques. They established a new approach in the investigation of these compounds based on high and low energy collision-activated dissociation.

Some of the analytical methods described in the literature rely on gas chromatographic-mass spectrometric analyses of derivatized coumarins [11]. Methods that employ direct gas chromatographic injections of untreated material also were proposed for selected compounds [12-14]. Liquid chromatographic methods are extensively used for the analysis of rather complex mixtures of different coumarins. These methods include thin layer chromatography (TLC) [15] and highperformance liquid chromatography (HPLC) methods with reversed-phase and direct-phase separations [16, 17]. TLC also is widely used for sample preparation and clean-up procedures for plant extracts. Alcock et al. [18] described an interesting and pioneering application of liquid chromatography-mass spectrometry analysis (LC/MS) of timbo powder extracts. They used a glass-lined stainless steel microbore column coupled to a mass spectrometer via a moving belt LC/MS interface.

Current LC/MS interfaces allow realization of the inherent potential of both techniques, which thus expands the application capabilities and greatly simplifies the overall analytical procedures. In particular, the 
particle beam (PB) interface [19-28] has proved valuable for LC/MS analysis of small molecules. It is fully compatible with electron impact ionization (EI) or chemical ionization (CI), with no significant constraints regarding the liquid chromatography requirements or analyte polarity, which makes it an invaluable technique for analyses of complex mixtures. The analysis of coumarins by LC-PB/MS may offer a valid method for evaluation of the presence of certain coumarins in specific plants or for monitoring variable concentrations when coumarins are used as pharmaceutical preparations.

Our research group recently has modified a particle beam interface to make it compatible with mobile phase flow rates as low as $1 \mu \mathrm{L} / \mathrm{min}$ [29-31]. This new coupling device is fully compatible with preexisting instrumentation and offers several advantages over a conventional interface: drastic reduction in solvent consumption with negligible contamination of the mass spectrometer by column effluent; wider choice of solvents and buffers that are potentially harmful to the instrument; better signal response for high water content mobile phases with improved sensitivity and chromatographic performance during gradient analyses; easier and more effective tunability of the interface for chemically different mobile phases and analytes.

In this work an LC-PB/MS method for the analysis of 18 different coumarins, based on a micro particle beam interface, is reported. A sample of Smyrnium perfoliatum L. (Umbelliferae) was extracted and analyzed with this method and several coumarins were identified and quantified. This species can be considered a typical example of coumarin-containing plants.

\section{Experimental}

\section{Particle Beam Interface and Mass Spectrometer}

A modified Hewlett-Packard (Palo Alto, CA) 59980B particle beam interface, coupled with a HewlettPackard 5989A quadrupole mass spectrometer, was employed. The original nebulizer was replaced by a laboratory-made micronebulizer, which has been described in a previous paper [29]. The new device does not require modifications to the desolvation chamber, momentum separator, or their original assembly. The final transfer tube prior to the ion source was in the fully retracted position. A $50-\mu \mathrm{m}$-i.d., $180-\mu \mathrm{m}$-o.d. fused silica capillary tubing (Polymicro Technologies, Phoenix, AZ) was used as the nebulizer tip and to connect the chromatographic column. The nebulizing gas was helium 5.6 purity grade $(>99.9996 \%)$ and was obtained from SOL (Milano, Italy). The helium flow rate was about $0.2 \mathrm{~L} / \mathrm{min}$ when maximum signal response was monitored by the ion source with a mobile phase flow rate of $2 \mu \mathrm{L} / \mathrm{min}$. This value corresponds to a gas pressure of $30 \mathrm{lb} / \mathrm{in}^{2}{ }^{2}$ and to a linear velocity at the nebulizer tip of $200 \mathrm{~m} / \mathrm{s}$. The gas temperature was ambient while the desolvation chamber tempera- ture was $40{ }^{\circ} \mathrm{C}$ for all the experiments. The pressure was reduced to about $0.5 \mathrm{~atm}$ in the desolvation chamber, 0.3 torr in the second stage of the momentum separator, and 5-8 $\times 10^{-5}$ torr in the manifold of the ion source. The ion source temperature was set at 250 ${ }^{\circ} \mathrm{C}$ and the analyzer was at $120^{\circ} \mathrm{C}$. The mass spectrometer tuning and calibration were performed automatically by using perfluorotributylamine as the reference compound. The repeller potential was adjusted manually to monitor fragment ions with mass-to-charge ratios close to sample values. The mobile phase was allowed into the ion source during calibration. The mass spectrometer was scanned from $\mathrm{m} / \mathrm{z} 50$ to 300 with a threshold of 50 counts. The scan speed was 1.2 scans per second, which gave a mean of about 10 acquisition samples for each HPLC peak. The complete mass selection report of the selected ion monitoring (SIM) program used in this work is listed in Table 1. With the exception of a few compounds, to maximize the dwell time, the identification criteria were based on one characteristic ion for each substance. Peak area values were calculated with automatic integration. The electron energy was set at $70 \mathrm{eV}$ in the positive ion mode.

Signal optimization with the new interface was less critical and consistently easier than with the conventional interface. A specific combination of the position of the fused silica capillary inside the coaxial gas

Table 1. SIM acquisition program for the analysis of coumarins

\begin{tabular}{cccc}
\hline Group & Channels & $m / z$ & $\begin{array}{c}\text { Dwell time } \\
\text { (ms) }\end{array}$ \\
\hline \hline 1 & 13 & 73.00 & 150 \\
& 208.00 & 150 \\
& 178.00 & 150 \\
& 202.00 & 150 \\
& 146.00 & 150 \\
& 148.00 & 150 \\
& 162.00 & 150 \\
& 222.00 & 150 \\
& & 192.00 & 150 \\
& & 206.00 & 150 \\
& & 150.00 & 150 \\
& & 120.00 & 150 \\
& & 118.00 & 150 \\
& & 176.00 & 250 \\
& & 173.00 & 250 \\
& & 246.00 & 250 \\
& & 186.00 & 250 \\
& & 160.00 & 250 \\
& & 216.00 & 250 \\
& & 206.00 & 250 \\
& & 231.00 & 250 \\
& & 174.00 & 900 \\
& & & 900 \\
& & &
\end{tabular}



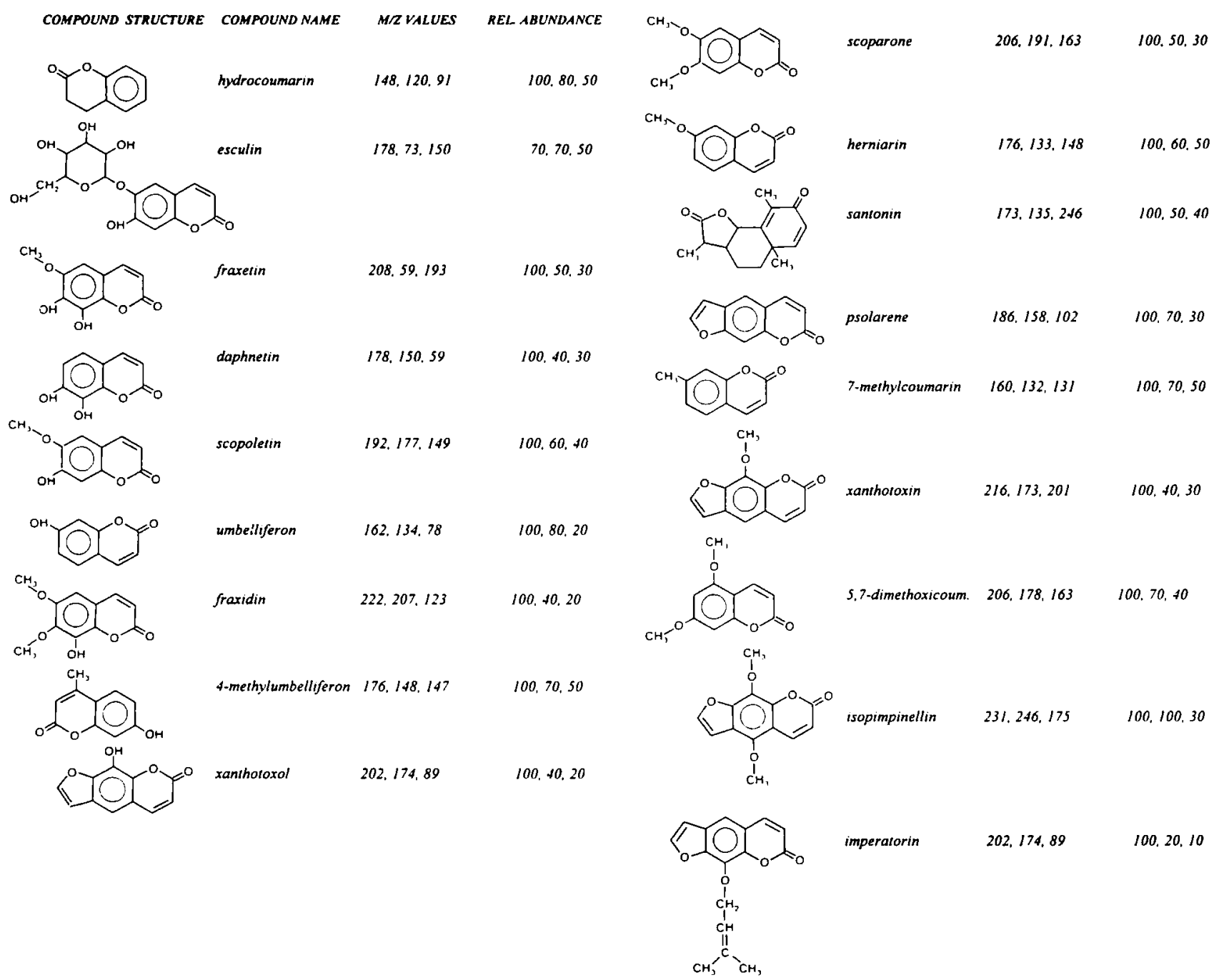

Figure 1. Coumarin structures with characteristic EI mass-to-charge ratio values and their relative intensities.

tubing and the helium pressure offered the highest signal intensity no matter what compound or mobile phase composition was used. This allowed reproduction of the same operating conditions throughout the chromatographic run. The optimization procedure was carried out with a flow injection analysis technique for preliminary adjustment, followed by HPLC mass spectrometry under the required chromatographic conditions. An important property of the new interface is the good performance even with high concentrations of water in the mobile phase. The signal response remained nearly constant over a wide range of mobile phase concentrations. Tuning, for example, was not affected by the water content in the mobile phase, which thus simplified the overall procedure. All the tuning tests were conducted with a $50 \%$ wateracetonitrile solution. A Vernier dial allowed 1/10-mm shifts of the nebulizer capillary tip; the most appropriate position was with the tip end aligned with the sharp restriction of the gas tubing or slightly withdrawn.

\section{Liquid Chromatography}

The packed capillary column used in this work was made in our laboratory from $1 / 16$-in. o.d., $250-\mu \mathrm{m}$ i.d. PEEK tubing (Alltech Associates Inc., Deerfield, IL) and was packed with 5- $\mu \mathrm{m}$ particle size reversed-phase C18 purchased from Phase Sep (Queensferry, UK) [32]. The 25-cm-long column had an efficiency of about 15,000 theoretical plates measured at $1 \mu \mathrm{L} / \mathrm{min}$ flow rate. Liquid chromatography was carried out with a Kontron 420 dual-pump binary-gradient conventional HPLC system (Kontron Instrument, Milano, Italy). Microliter flow rates were obtained with a laboratorymade splitter that was placed between the pumping system and the injector [33, 34]. The splitter allowed accurate and stable micro flow rates and rapid delivery 
of solvent concentration changes for reliable and reproducible gradients. A motor-assisted solvent mixer was placed after the pumps and before the splitter device. For sample injection, a zero-volume Valco injector equipped with a $60-\mathrm{nL}$ internal loop was employed (Valco, Houston, TX). Larger loops are not advisable for flow rates lower than $5 \mu \mathrm{L} / \mathrm{min}$ because of loss of chromatographic efficiency.

Stock solutions were prepared by dissolving $10 \mathrm{mg}$ of each compound in $1 \mathrm{~mL}$ of methanol $(10,000 \mathrm{ppm})$. The separation of the coumarin mixture was optimized with a UV detector equipped with a micro flow cell. For the chromatographic separation of the coumarins, a mixture of water and acetonitrile was used as the mobile phase. The mobile phase flow rate was set at 2 $\mu \mathrm{L} / \mathrm{min}$. The acetonitrile relative concentration was kept $5 \mathrm{~min}$ at $0 \%$, sharply increased to $40 \%$, and then increased from 40 to $80 \%$ in $25 \mathrm{~min}$ for the gradient run. The initial isocratic step achieved solute band focusing and improved separation efficiency.

All solvents were HPLC grade from Farmitalia Carlo Erba (Milano, Italy) and were filtered and degassed before use. Reagent water was obtained from a Milli-Q water purification system (Millipore Corp., Bedford, MA).

\section{Extraction Procedure}

Fresh plant $(0.150 \mathrm{~g})$ was extracted by manual shaking with $10 \mathrm{~mL}$ of methanol. The crude extract was concentrated under vacuum $\left(40^{\circ} \mathrm{C}\right)$ to $0.5 \mathrm{~mL}$. The concentrated extract was separated by TLC (silica gel 60; eluent: toluene-diethyl ether-acetic acid 50:45:5). The zone from start to $R_{f}=0.6$ was scraped and the gel was extracted with methanol. This first cleanup eliminated chlorophyll pigments from the extract. After vacuum concentration the extract was further separated by TLC (silica gel 60; eluent: chloroformmethanol 85:15). The zone from $R_{f}=0.2$ to front was scraped and the gel was extracted with methanol. Finally the methanolic extract was filtered $(0.2 \mu \mathrm{m})$ and concentrated to $100 \mu \mathrm{L}$. Smyrnium perfoliatum $\mathrm{L}$. was collected from cultivated plants in the garden at the Institute of Botany, University of Urbino, Italy. Voucher specimens have been deposited in the herbarium of the Institute of Botany, Urbino University, Italy (S.P. 1-93).

\section{Results and Discussion}

A number of different coumarins were analyzed during this study. All of them were suitable for reversedphase liquid chromatography and gave reproducible and interpretable EI mass spectra. Figure 1 shows the structural formulas of the 18 components, three characteristic EI mass spectral fragments, and their relative intensities. A solution of water and acetonitrile was used as the mobile phase. Despite a slightly better overall performance with methanol, which gave a higher signal response with the particle beam interface, it was rejected because of its higher viscosity. The micro-HPLC assembly is particularly vulnerable to very high operating pressure especially at the connection points, where fused silica capillary tubing is held in place by weak plastic ferrules. Moreover a mixture of water and acetonitrile usually gave a more stable and constant response during a common gradient run. To confirm these results a specific test was performed. Figure 2 shows the system signal response versus variations in the mobile phase composition: plot a is obtained with a combination of methanol and water, whereas plot $b$ is obtained with acetonitrile and water. Sixty nanograms of imperatorin was injected five times for each mobile phase composition. The two curves show similar effects of solvent on the ion signal. Characteristic and critical relationships between substances and mobile phases are part of the particle beam performance. The performance of the interface is strongly dependent on the choice of the mobile phase and on the structure of the analyte. Imperatorin was chosen arbitrarily and the results safely may be extended for similar compounds. Chromatographic separation of coumarins was performed with a $\mathrm{C} 18$ reversed-phase packed capillary column. The $250-\mu \mathrm{m}$ i.d. columns
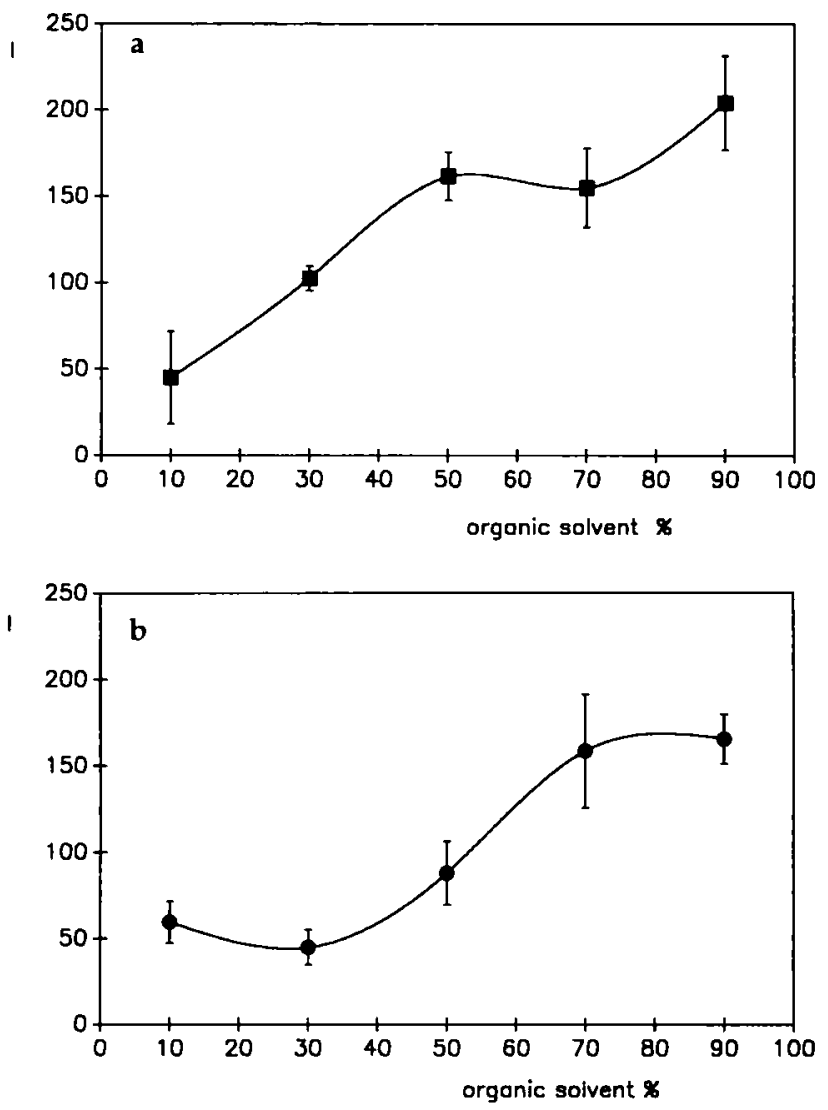

Figure 2. Instrument signal response ( $l$ ) versus variation in mobile phase composition: (a) methanol and water; (b) acetonitrile and water. 


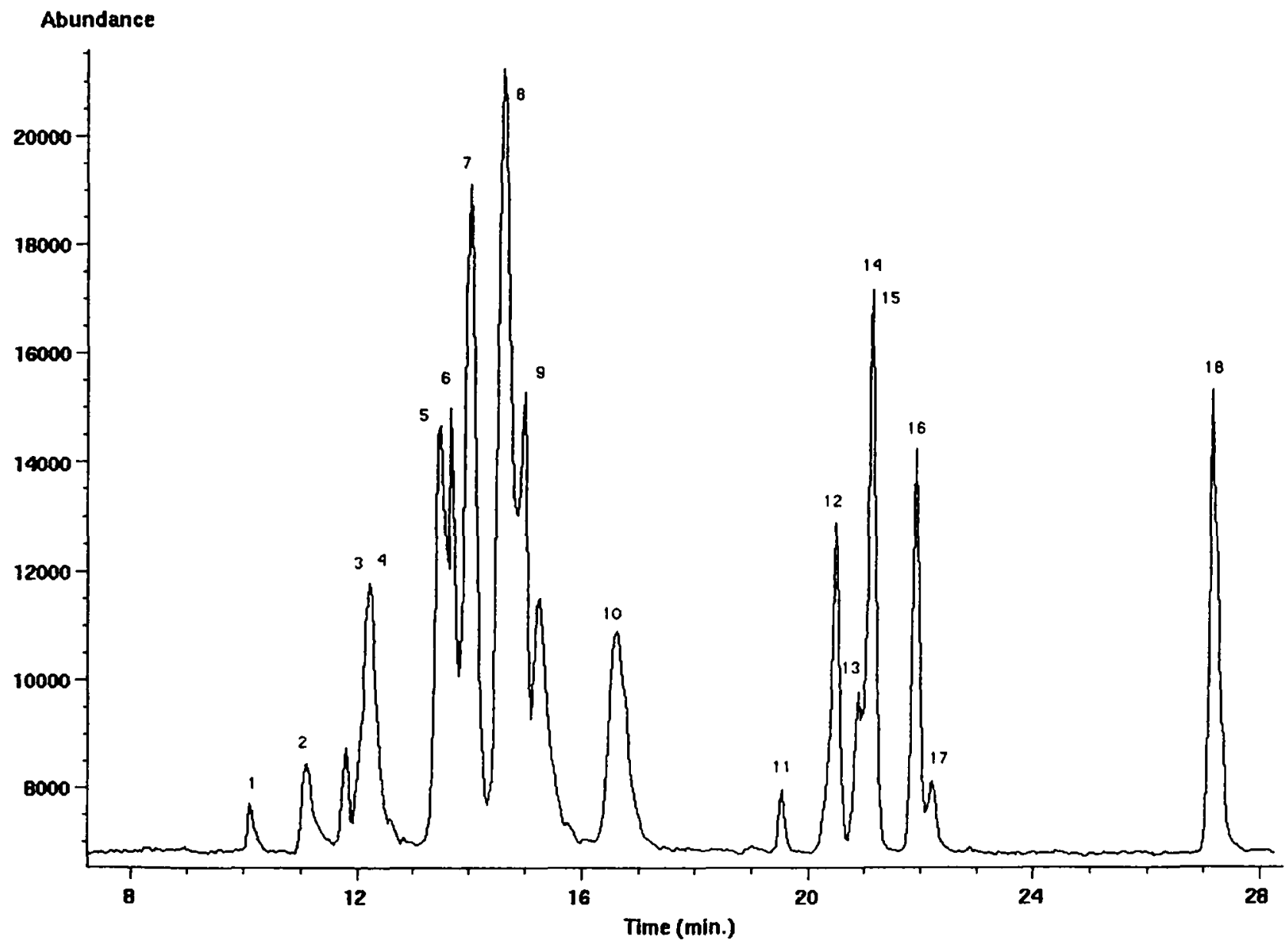

Figure 3. Reconstructed ion chromatogram of coumarin standard mixture: (1) hydrocoumarin; (2) esculin; (3) fraxetin; (4) daphnetin; (5) scopoletin; (6) umbelliferon; (7) fraxidin; (8) 4-methylumbelliferon; (9) xanthotoxol; (10) scoparone; (11) herniarin; (12) santonin; (13) psolarene; (14) 7-methylcoumarin; (15) xanthotoxin; (16) 5,7-dimethoxicoumarin; (17) isopimpinellin; (18) imperatorin. The area counts intensity is reported on the $y$ axis.

performed best with a mobile phase flow rate of about $2 \mu \mathrm{L} / \mathrm{min}$. The lowest height of the reduced theoretical plate $h$ is obtained with a reduced linear velocity $v$ 1.87 , which corresponds to a mobile phase flow rate of $1 \mu \mathrm{L} / \mathrm{min}$. Reduced chromatographic parameters, such as $h$ and $v$, were introduced several years ago by Bristow and Knox [35] to allow comparison among columns packed with different materials and packing methods, and when eluents of different viscosities or solutes of different diffusion coefficients were used. In fact, for example, the plate height $(H)$ is expressed in terms of particle diameter units $\left(d_{p}\right), h=H / d_{p}$; the eluent velocity $(u)$ is expressed relative to the rate of diffusion across a particle $\left(D_{\mathrm{m}}\right), v=u d_{\mathrm{p}} / D_{\mathrm{m}}$. These parameters are dimensionless and allow ready comparison of results obtained in different experimental conditions. Slightly higher flow rates can speed up the analysis with a negligible loss of chromatographic efficiency. This situation is due to a flatter van Deemter curve around the lowest flow rates. The splitter device adopted for the micro flow rate generation guarantees a linear and reproducible gradient. For a correct identification, precise and reliable retention times are essential. The micro-HPLC system assured retention time variations lower than $\pm 1 \%$ for a retained compound during a gradient analysis [34]. Compound transfer delay between column output and the appearance of a mass spectrometric signal was less than $1 \mathrm{~min}$. This travel time does not influence the chromatographic band shape and does not compromise separation efficiency even for critical separations.

Figure 3 shows a reconstructed ion chromatogram obtained from a SIM analysis of the coumarin standard mixture with amounts that ranged between 30 and 100 ng. Figure 4 shows the SIM traces that correspond to some partially overlapped peaks eluted between 13:00 and 16:00 min. All signal interferences were virtually eliminated. Variable signal response was observed with different coumarins. Table 2 shows detection limits for the 18 coumarins analyzed with the program described previously. The limits were determined by analysis of the specified quantity and for a signal-to-noise ratio of 
Table 2. Instrument detection limits for the 18 coumarins considered; signal-to-noise ratio 5:1

\begin{tabular}{lc}
\hline Compound & $\begin{array}{c}\text { Quantity } \\
\text { (ng) }\end{array}$ \\
\hline \hline 1. Hydrocoumarin & 2.0 \\
2. Esculin & 50.0 \\
3. Fraxetin & 70.0 \\
4. Daphnetin & 40.0 \\
5. Scopoletin & 7.0 \\
6. Umbelliferon & 1.5 \\
7. Fraxidin & 1.5 \\
8. 4-Methylumbelliferon & 1.0 \\
9. Xanthotoxol & 4.0 \\
10. Scoparone & 1.0 \\
11. Herniarin & 3.0 \\
12. Santonin & 1.5 \\
13. Psoralene & 0.7 \\
14. 7-Methylcoumarin & 5.0 \\
15. Xanthotoxin & 0.7 \\
16. 5.7-Dimethoxicoumarin & 1.0 \\
17. Isopimpinellin & 3.0 \\
18. Imperatorin & 0.6
\end{tabular}

5:1. Calibration and injection reproducibility were checked with the method proposed by using imperatorin. Each substance was injected five times in different amounts that ranged between 1 and $60 \mathrm{ng}$ to be consistent with the actual sample concentration. Each sample was introduced without the column, directly into the mass spectrometer via the particle beam interface and with a mobile phase composed of an equal concentration of water and acetonitrile. The equation relative to the linear regression plot for the concentration calibration is $y=35.2 x-34.1$ with a correlation coefficient of 0.998 . The mean standard deviation, calculated by using the average of the peak area values for each concentration experiment, was $\pm 10.5 \%$. The mass spectrometer was operated in SIM mode at $\mathrm{m} / \mathrm{z}$ 202. The mobile phase was kept at $2 \mu \mathrm{L} / \mathrm{min}$ constantly for all experiments. Excellent linearity can be observed in the calibration plot and for the concentration range considered. The addition of buffers, usually employed to improve the particle beam carrier mechanism and consequently the response linearity, was not considered in this case. Different neutral $\mathrm{pH}$ buffers were tested (i.e., ammonium acetate and potassium

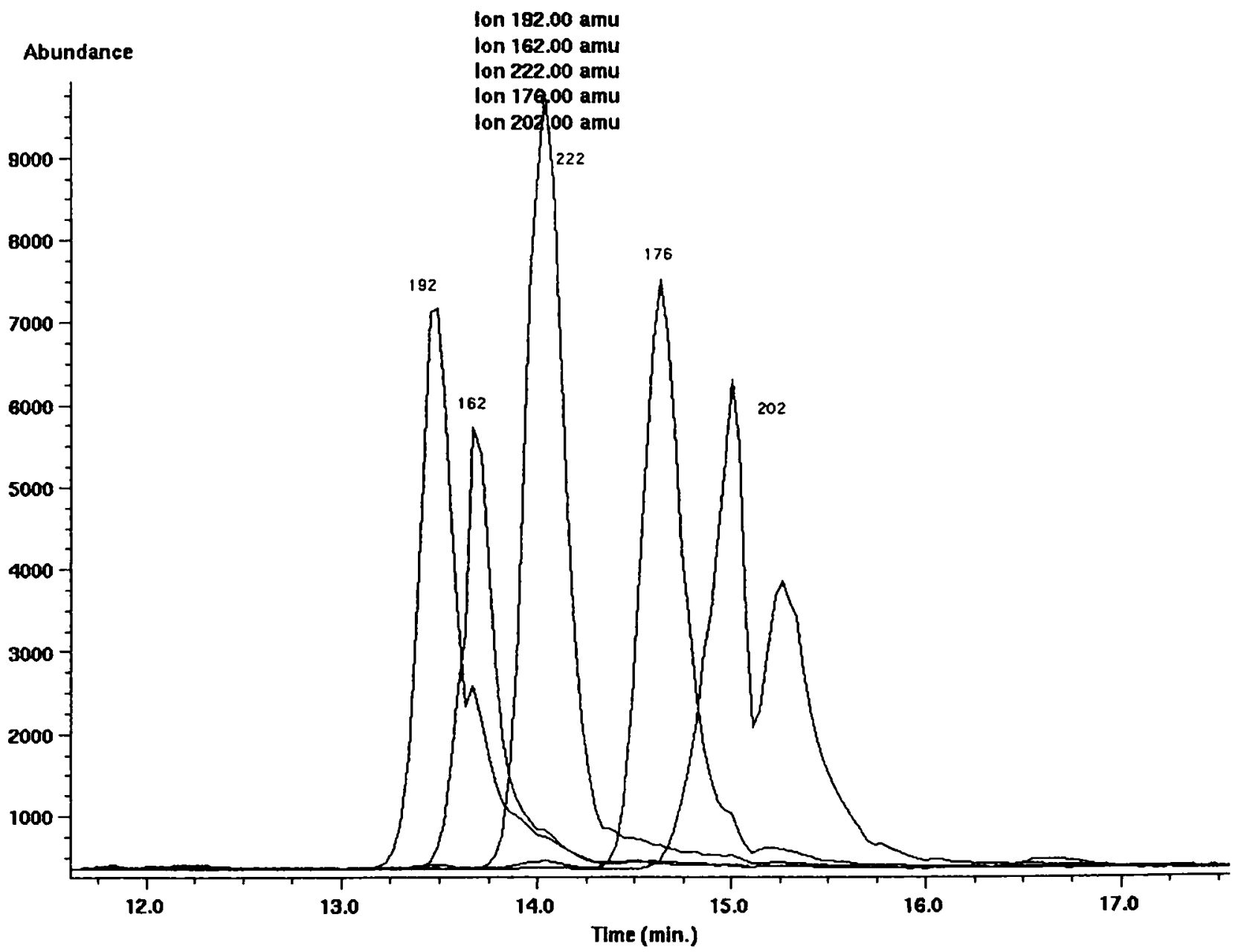

Figure 4. Ion chromatograms relative to scopoletin $(m / z 192)$, umbelliferon $(m / z 162)$, fraxidin $(m / z 222), 4$-methylumbelliferon $(m / z 176)$, and xanthotoxol $(m / z 202)$. 


\section{Abundance}

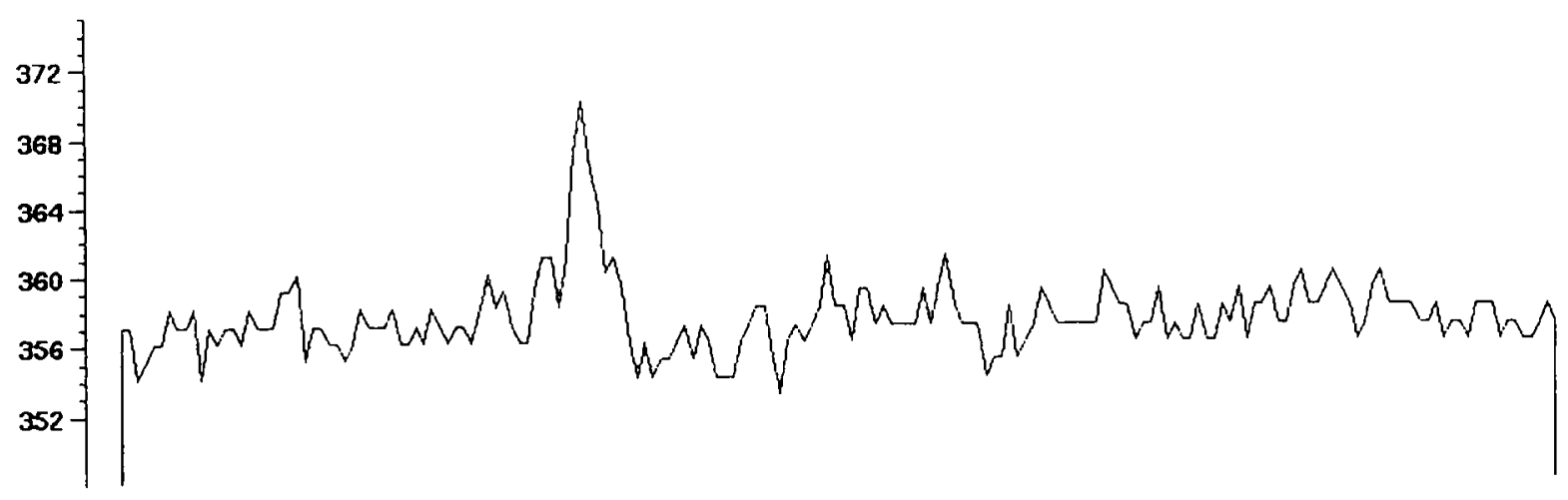

Figure 5. SIM trace at $m / z 216$ (xanthotoxin) obtained from the analysis of plant extract.

dihydrogen orthophosphate), but they all reduced chromatographic resolution. Furthermore, linearity for the instrument response was excellent in that concentration range and any buffer addition was unnecessary. The addition of nonvolatile buffers is tolerated by the instrument because of the very low liquid intake [31].

Methanol plant extracts were concentrated to 100 $\mu \mathrm{L}$ and injected into the system. Identification criteria were based on retention times and specific responses to characteristic mass-to-charge ratio values. Because of the extremely low concentration of the compounds detected in the extract, the instrument sensitivity was enhanced by selecting the lowest number of characteristic ions and the highest dwell time for a given program. Most of the compounds were thus identified by using only the highest characteristic ion. The consequent loss of detection specificity was balanced by an additional cleanup step as described in the Experimental section. Quantitation was obtained by direct comparison between analyte peak areas and a conveniently diluted standard solution of each coumarin. We assumed, for each coumarin, a linear response slightly above the detection limit as shown by the results obtained from the imperatorin calibration. The results are summarized in Table 3. The confidence level of the presented results was extrapolated from the mean standard deviation calculated for the calibration curve

Table 3. Amounts and sample concentrations relative to detected coumarins in Smy/rnium perfoliatum L. plant extract

\begin{tabular}{lcc}
\hline Compound & $\begin{array}{c}\text { Quantity } \\
\text { (ng) }\end{array}$ & $\begin{array}{c}\text { Sample } \\
\text { conc. }(\mu \mathrm{g} / \mathrm{g})\end{array}$ \\
\hline \hline 4-Methylumbelliferon & 0.7 & 7.3 \\
Xanthotoxol & 3.8 & 40.3 \\
Scoparone & 1.0 & 10.6 \\
Xanthotoxin & 0.6 & 6.3 \\
Fraxidin & 1.0 & 10.6 \\
\hline
\end{tabular}

$( \pm 10.5 \%)$. Figure 5 shows the SIM trace relative to an identified coumarin (xanthotoxin).

Some of the amounts calculated were equal to or lower than the corresponding detection limits. In particular, scoparone and xanthotoxol gave signal responses close to the signal-to-noise ratio considered for the evaluation of the detection limits (5:1) and consequently their detection limits correspond to the calculated amounts in the sample. Although all other compounds fall slightly below these limits, their amounts have been estimated by a careful comparison of the peak areas with those obtained from the diluted standard solution.

This method can be considered as a valid aid in the investigation of the composition of complex matrices such as plant extracts. Coumarins are a very important group of active substances, and better knowledge of their presence in biological samples is always desirable.

\section{References}

1. Ceska, O.; Chaudhary, S. K.; Warrington, P. J.; AshwoodSmith, M. J. Phytochemistry 1987, 26, 165-169.

2. Rodighiero, G.; Dall'Acqua, F.; Pathak, M. A. In Topics in Photomedicine; Smith, K. C., Ed.; Plenum: New York, 1984; p 319.

3. Murray, R. D. H.; Mendez, J.; Brown, S. A. In The Natural Coummins; Wiley: New York, 1982; pp 45-53.

4. Harrison, A. G. Chemica! lonisation Mass Spectrometry; CRC Press, Boca Raton, FL, 1983.

5. Plattner, R. D.; Spencer, G. F. Org. Mass Spectrom. 1988, 23 , 624.

6. Voight, D. I. Prakt. Chem. 1977, 319. 767.

7. Evans, C.; Traldi, P.; Chilin, A.; Pastorini, G.; Rodighiero, P. Org. Mass Spectrom. 1991, 26, 688-694.

8. Bravo, P.; Ticozzi, C.; Daolio, S.; Traldi, P. Org. Mass Spectrom. 1985, 20, 740-747.

9. Pelli, B.; Traldi, P.; Rodighiero, P.; Guiotto, A. Biomet. Envirom. Mass Spectrom. 1986, 13, 417-422. 
10. Horning, S. R.; Bier, M. E.; Cooks, R. G.; Brusini, G.; Traldi, P.; Guiotto, A.; Rodighiero, P. Biomed. Environ. Mass Spectrom. 1989, 18, 927-934.

11. De Vries, J. X.; Taucher, B.; Wurzel, G. Biomed. Environ. Mass Spectrom. 1988, 15, 413-417.

12. Yamamoto, K.; Kato, S.; Shimomura, H. I. Clromatogr. 1986, $362,274-277$

13. Marles, R. J.; Compadre, C. M.; Farnsworth, N. R. Econ. Bot. 1987, 41, 41-47.

14. Weinberg, D. S.; Manier, M. L.; Richardson, M. D.; Haibach, F. G. I. Agric. Food Chem. 1993, 41, 48-51.

15. Glowniak, K.; Matysik, G.; Bieganowska, M.; Soczewinski, E. Chromatographin 1986, 22, 307-310.

16. Vande Casteele, K.; Geiger, H.; Van Sumere, C. F. I. Chromatogr. 1983, 258, 111-124.

17. Zogg, G. C.; Nyiredy, Sz.; Sticher, O. Cltromatographia 1989, 27, 591-595.

18. Alcock, N. J.; Corbelli, L.; Games, D. E.; Lant, M. S.; Westwood, S. A. Biomed. Mass Spectrom. 1982, 9, 499-504.

19. Yinon, J.; Jones, T. L.; Betowski, L. D. J. Chromatogr. 1989, $482,75-85$.

20. Girault, J.; Istin, B.; Malgouyat, J. M.; Brisson, A. M.; Fourtillan, J. B. /. Chromatogr. 1991, 564, 43-53.

21. In Suk Kim; Sasinos, F. I.; Stephens, R. D.; Brown, M. A. J. Agric. Food Chem. 1990, 38, 1223-1226.
22. Jones, T. L.; Betowski, L. D.; Lesnik, B.; Chiang, T. C.; Teberg, J. E. Environ. Sci. Technol. 1991, 25, 1880-1884.

23. Jones, G. G.; Pauls, R. E.; Willoughby, R. C. Anal. Chem. 1991, $63,460-463$.

24. Julien-Larose, C.; Voirin, P.; Mas-Chamberlin, C.; Dufour, A. I. Chromatogr. 1991, 562, 39-45.

25. Hsu, J. Anal. Chem. 1992, 64, 434-443.

26. Bagheri, H.; Slobodnik, J.; Marce Recasens, R. M.; Ghijsen, R. T.; Brinkman, U. A. Th. Cltromatographin 1993, 37, 159-167.

27. Voyksner, R. D.; Straub, R.; Keever, J. Environ. Sci. Technol. 1993, 27, 1665-1672.

28. Behymer, T. D.; Bellar, T. A.; Budde, W. L. Anal. Chem. 1990 $62,1686-1690$

29. Cappiello, A.; Bruner, F. Anal. Chem. 1993, 65, 1281-1287.

30. Cappiello, A.; Famiglini, G.; Bruner, F. Anal. Chem., 1994, 66, 1416-1423.

31. Cappiello, A.; Famiglini, G. Anal. Chem., 1994, 66, 3970-3976.

32. Cappiello, A.; Palma, P.; Mangani, F. Chromatographia 1991, $32,389-391$

33. Palma, P.; Cappiello, A. Ann. Chimica 1992, 82, 371-377.

34. Berloni, A.; Cappiello, A.; Famiglini, G.; Palma, P. Cliromatographia, submitted.

35. Bristow, P. A.; Knox, J. H. Chromatographia 1977, 10, 279-289. 